\title{
O fenômeno da fibromialgia: prevalência de problemas psíquicos nos pacientes portadores da doença
}

\author{
The phenomenon of fibromyalgia: prevalence of psychological problems in patients \\ with the disease
}

El fenómeno de la fibromialgia: prevalencia de problemas psicológicos en pacientes con la enfermedad

Thais Oliveira Leite ${ }^{1 *}$, Luana Maria Libório da Mota1, Lorena Vieira Fernandez de Araújo ${ }^{1}$, Diêgo Andrade de Oliveira ${ }^{1}$, Aline Reis Freitas ${ }^{1}$, Dandara Meira dos Reis ${ }^{1}$, Tenylle Botelho Fernandes ${ }^{1}$, João Pedro Moraes Borges ${ }^{1}$, Luana Alves Lisboa ${ }^{1}$, Rosângela Souza Lessa1 .

\section{RESUMO}

Objetivo: Descrever a prevalência de sintomas depressivos de pacientes com Fibromialgia em uma cidade do interior da Bahia. Métodos: Trata-se de um estudo de corte transversal, com abordagem quantitativa, com 71 fibromiálgicos. A coleta de dados ocorreu por meio de um questionário on-line composto por perguntas sobre perfil sociodemográfico e o Inventário de Depressão de Beck (BDI). O estudo foi aprovado por Comitê de Ética em Pesquisa. Resultados: Obteve-se dessa amostra um predomínio do sexo feminino (98,6\%), na faixa etária de 31 a 50 anos (74,6\%), 57,7\% eram pardas, $64,8 \%$ casada ou em união estável e $52,1 \%$ com nível superior. Quanto às atividades remuneradas, $62 \%$ responderam estar trabalhando. Em relação à prática de atividade física, 36,6\% afirmaram não realizar. No BDI, 49,3\% foram classificados com depressão moderada a grave, indicando elevado comprometimento de sintomas psíquicos. Conclusão: A interferência negativa da Fibromialgia na qualidade de vida do fibromiálgico devido às manifestações clínicas da doença é evidente, principalmente relacionado às limitações físicas e emocionais. Diante disso, são necessários estudos mais aprofundados para entender as limitações impostas pela doença, processo esse que pode ser facilitado por profissionais da saúde e da sua rede familiar.

Palavras-chave: Fibromialgia, Depressão, Dor crônica, Reumatologia.

\begin{abstract}
Objective: Describe the prevalence of depressive symptoms in patients with fibromyalgia in a city in the interior of Bahia. Methods: This is a cross-sectional study, with a quantitative approach, with 71 patients with fibromyalgia. Data collection took place through an online questionnaire consisting of questions about sociodemographic profile and the Beck Depression Inventory (BDI). The study was approved by the Research Ethics Committee. Results: From this sample, there was a predominance of females (98.6\%), aged between 31 and 50 years $(74.6 \%), 57.7 \%$ were brown, $64.8 \%$ married or in union stable and $52.1 \%$ with higher education. As for paid activities, $62 \%$ said they were working. Regarding the practice of physical activity, 36 $.6 \%$ said they did not do it. In the BDI, $49.3 \%$ were classified as having moderate to severe depression, indicating high impairment of psychic symptoms. Conclusion: The negative interference of fibromyalgia in the quality of life of fibromyalgia patients due to the clinical manifestations of the disease is evident, mainly related to physical and emotional limitations. Therefore, further studies are needed to understand the limitations imposed by the disease, a process that can be facilitated by health professionals and their family network.
\end{abstract}

Keywords: Fibromyalgia, Depression, Chronic pain, Rheumatology.

${ }^{1}$ Faculdades Santo Agostinho, Vitória da Conquista - BA. *E-mail: thaisoleite@hotmail.com

SUBMETIDO EM: 10/2021

ACEITO EM: 10/2021

PUBLICADO EM: 11/2021 


\section{RESUMEN}

Objetivo: Describir la prevalencia de síntomas depresivos en pacientes con fibromialgia en una ciudad del interior de Bahía. Métodos: Se trata de un estudio transversal, con abordaje cuantitativo, con 71 pacientes con fibromialgia. La recogida de datos se realizó a través de un cuestionario online compuesto por preguntas sobre perfil sociodemográfico y el Inventario de Depresión de Beck (BDI). El estudio fue aprobado por el Comité de Ética en Investigación. Resultados: De esta muestra, hubo predominio del sexo femenino (98,6\%), con edades comprendidas entre 31 y 50 años $(74,6 \%$ ), el $57,7 \%$ eran morenos, el $64,8 \%$ casados o en unión estable y el $52,1 \%$ con estudios superiores. En cuanto a las actividades remuneradas, el $62 \%$ dijo estar trabajando. En cuanto a la práctica de actividad física, el $36,6 \%$ dijo no realizarla. En el BDI, el $49,3 \%$ se clasificó como con depresión moderada a grave, lo que indica un alto deterioro de los síntomas psíquicos. Conclusión: Es evidente la interferencia negativa de la fibromialgia en la calidad de vida de los pacientes con fibromialgia debido a las manifestaciones clínicas de la enfermedad, principalmente relacionada con limitaciones físicas y emocionales. Por tanto, son necesarios más estudios para comprender las limitaciones que impone la enfermedad, proceso que puede ser facilitado por los profesionales sanitarios y su red familiar.

Palabras clave: Fibromialgia, Depresión, Dolor crónico, Reumatología.

\section{INTRODUÇÃO}

A dor é um fenômeno de sensibilização central que afeta cronicamente as pessoas com fibromialgia, causando impactos econômicos e sociais em todo o mundo. As dores crônicas são reconhecidas como um grave problema de saúde pública, uma vez que a literatura aponta que a mesma ocasiona prejuízos tanto em níveis físicos, bem como emocionais, gerando custos aos cofres públicos em países desenvolvidos e nos com baixos índices de desenvolvimento econômico (SOUZA JB e PERISSINOTTI DMN, 2018).

A Fibromialgia (FM) é uma doença crônica, de etiologia multifatorial cujo principal sintoma é a dor física difusa, de característica variável, desde uma sensação de queimação, pontada, peso, "cansaço" ou como contusão, podendo ser agravada por mudança climática, tensão emocional ou por esforço físico (ARAUJO ABM, 2020).

A fisiopatologia da FM associa-se a um mecanismo de sensibilização do sistema nervoso central (SNC), o qual provavelmente resulta de uma disfunção de neurotransmissores. Tal distúrbio incluiria uma deficiência de neurotransmissores supressores da dor (serotonina, norepinefrina), ou uma hiperatividade dos neurotransmissores excitatórios, como a substância P e o glutamato (FRIEDRICH JV, et al., 2020).

Os portadores da FM, além da dor generalizada, apresentam vários outros sintomas incapacitantes, como rigidez matinal, fadiga crônica, distúrbios do sono, cefaleia, transtornos comportamentais, depressão, ansiedade, deficiência de memória, desatenção, tontura, vertigens, parestesias, sintomas compatíveis com síndrome do intestino irritável (SII) ou com a síndrome das pernas inquietas. Os sintomas variam de leves a intensos, prejudicando diretamente as atividades de vida diária e a qualidade de vida do paciente, que passa a viver em função da doença e do tratamento (FARIA PC, et al., 2014).

Diante da variedade do quadro clínico e de até o momento não existir marcador laboratorial ou exame de imagem característico, o diagnóstico da FM é eminentemente clínico, baseado na contagem e pesquisa dos pontos dolorosos (tender points) no corpo do paciente (HEYMANN RE, et al., 2017).

Quanto ao tratamento da FM, ele pode ser dividido em terapia farmacológica e não farmacológica. Os antidepressivos tricíclicos geralmente é um dos farmácos de escolha e a Terapia Cognitiva-Comportamental (TCC) atua auxiliando o pensamento e a forma pelo qual o paciente age perante os acontecimentos do dia a dia (ANDRADE ICS, 2017).

Este trabalho busca contribuir com os estudos sobre o tema, já que existe lacuna na literatura acerca da saúde mental em fibromiálgicos, para auxiliar na evolução do constante conhecimento sobre a doença e 
principalmente com a intenção de buscar formas que possam ajudar a aliviar sintomas físicos e psicológicos dos pacientes portadores. Assim, o presente estudo objetivou descrever a prevalência de sintomas depressivos de pacientes com Fibromialgia em uma cidade do estado da Bahia.

\section{MÉTODOS}

Trata-se de uma pesquisa epidemiológica, de corte transversal, com abordagem quantitativa, que buscou investigar as condições de saúde e qualidade de vida em pessoas com fibromialgia. A população foi composta por pacientes do sexo feminino e masculino, de forma probabilística, com idade entre 30 e 60 anos de idade, acompanhados em serviços de saúde, que já possuíam o diagnóstico médico confirmado da doença. Os mesmos foram convidados a participar da pesquisa por meio da divulgação nas redes sociais, por cartazes fixados em Unidades de Saúde da Família, em consultórios reumatológicos e em clínicas de fisioterapia do município.

A coleta de dados foi realizada por meio de um questionário/formulário via e-mail ou WhatsApp para os pacientes que previamente se voluntariaram a participar da pesquisa, sendo utilizado o aplicativo gratuito Google Forms para produção, envio e contabilização dos dados desses formulários virtuais. Neste formulário virtual constou os objetivos, as possíveis dúvidas sobre o processo de coleta de dados e o Termo de Consentimento Livre e Esclarecido (TCLE), que ressalta os aspectos éticos envolvidos e os riscos e benefícios da pesquisa (resolução 466/2012). O estudo foi aprovado pelo Comitê de Ética em Pesquisa da Faculdade de Medicina de Itajubá (FMIT), sob o parecer 4.036.120, tendo a coleta iniciada somente após aprovação.

Posteriormente, um bloco de perguntas para análise do perfil sociodemográfico foi aplicado, no qual perguntou-se a idade, cor da pele, sexo biológico, estado civil, escolaridade e detalhes sobre a ocupação profissional. Além disso, utilizou-se o Inventário de Depressão de Beck (BDI), a fim de autoavaliar sintomas de depressão. O Inventário é amplamente usado tanto em pesquisa como em clínica, tendo sido traduzido para vários idiomas, inclusive o português, e validado em diferentes países (BECK AT, et al., 1982; GORESTEIN C e ANDRADE L, 1998).

O Inventário de Depressão de Beck é composto por uma escala de 21 itens, no qual o entrevistado autorrelata suas respostas. Cada pergunta contém quatro opções de respostas afirmativas, com graus crescentes de intensidade de sintomas depressivos, e escores variando de 0 a 3 (BECK AT, et al., 1982; CUNHA JA, 2001).

Os itens do BDI foram aferidos por meio dos autorrelatos dos respondentes em relação aos 21 questionamentos realizados objetivamente. Assim, as perguntas tinham relação com sentimentos de tristeza, fracasso, culpa, prazer, punição, decepção pessoal, inferiorização, desânimo do futuro. Além do choro, irritabilidade, ideação suicida, desinteresse por contato com pessoas, dificuldades na tomada de decisão, baixa produtividade no trabalho, dificuldades com o sono, cansaço, falta de apetite, perda do peso, despreocupação com a saúde e aparência e ausência de interesse sexual (CASTRO SMJ, et al., 2010).

Estimou-se os sintomas depressivos no presente estudo por meio do escore total das 21 perguntas respondidas, variando de 0 a 63 pontos. A classificação se deu em quatro tipos de acordo com a intensidade dos sintomas referidos, em: mínima (0 - 11), leve (12 - 19), moderada (20 - 35) e grave (36 - 63) (CASTRO SMJ, et al., 2010).

Os dados quantitativos foram tabulados no Microsoft Excel $\circledast$ e analisados utilizando o programa estatístico software R versão 3.0.3 (2014-03-06), Copyright (C) 2014 The R Foundation for Statistical Computing, a fim de evidenciar os sintomas depressivos entre os participantes do estudo.

\section{RESULTADOS E DISCUSSÃO}

De acordo com o perfil sociodemográfico traçado pela amostra de 71 pessoas com fibromialgia participantes deste estudo, houve uma predominância de acometimento no sexo feminino $(98,6 \%)$ e de indivíduos na faixa etária de 31 a 50 anos de idade (74,6\%). A maior parte dos respondentes declarou ser pardo $(57,7 \%)$, casado ou em união estável $(64,8 \%)$ e ter cursado o ensino superior $(52,1 \%)$ (Tabela 1$)$. 
Tabela 1 - Perfil sociodemográfico dos fibromiálgicos $(n=71)$.

\begin{tabular}{lcc}
\hline Variáveis & $\mathbf{n}$ & $\%$ \\
\hline Idade (anos) & 4 & 5,6 \\
\hline $26-30$ & 53 & 74,6 \\
$31-50$ & 14 & 19,7 \\
$51+$ & & 98,6 \\
\hline Sexo & 70 & 1,4 \\
\hline Feminino & 1 & \\
Masculino & & 57,7 \\
\hline Cor da pele & 41 & 22,5 \\
\hline Parda & 16 & 15,5 \\
Branca & 11 & 1,4 \\
Preta & 1 & 2,8 \\
Indígena & 2 & 87,3 \\
Amarela & & 12,7 \\
\hline Estado civil & 62 & \\
\hline Casado ou união estável & 9 & 11,3 \\
Divorciado & & 36,6 \\
\hline Escolaridade & 8 & 52,1 \\
\hline Ensino fundamental completo e incompleto & 26 & \\
Ensino médio completo e incompleto & 37 & \\
Ensino superior completo, incompleto e pós graduação & & \\
\hline Filhos & 60 & \\
\hline Sim & 11 & \\
Não & & \\
\hline Fonte: Leite TO, & & \\
\hline
\end{tabular}

Fonte: Leite TO, et al., 2021.

Esses achados corroboram com Souza JB e Perissinotti DMN (2018), no qual afirmam que a prevalência da FM na população brasileira é de $2 \%$ com proporção de 1 homem para cada 5,5 mulheres, evidenciado pelo maior percentual de mulheres no presente estudo. Para Ramiro F de S, et al. (2013), as mulheres acometidas pela fibromialgia apresentam uma mudança negativa em relação à qualidade de vida, levando-as a níveis muito altos de estresse, o que ocorre, em parte, por conta do fator histórico do papel da mulher na sociedade e também por fatores biológicos característicos do sexo feminino, como cuidados domésticos, com a família, período pré-menstrual e desregulação hormonal. Além disso, a literatura aponta também uma grande dificuldade entre as pacientes com fibromialgia em identificar suas próprias emoções, somando-se a isso um número maior de queixas agravando a questão da dor e prejudicando a mobilidade.

Em relação ao fator idade, Rodrigues GF, et al. (2016), considera que a fibromialgia acomete pacientes em idade produtiva (de 30 a 50 anos), ou seja, idade produtiva, cuidar da casa, e de si mesmo e que, quando diagnosticados, essas tarefas corriqueiras já não podem mais ser realizadas ou, quando realizadas, exigem um esforço maior do paciente, diminuindo diretamente a qualidade de vida destes.

Não é possível inferir conclusões quanto à distribuição racial da FM, aja vista que é uma doença que acomete todas as raças. No que se refere aos dados de estado civil, observa-se que este corrobora aos achados do estudo de Melo GA, et al. (2019), no qual a maioria também eram casadas. Quanto à escolaridade, não houve uma prevalência unânime na literatura.

Em relação às atividades remuneradas, 38\% dos respondentes relataram não realizar nenhuma e, portanto, não possuir renda própria. Dentre os que responderam possuir um emprego (62\%), a maioria cumpria uma carga horária de trabalho semanal de 20 a 30 horas $(81,7 \%)$ e trabalhavam pelo menos dois 
turnos ao dia (46,5\%). Além disso, o estudo foi composto majoritamente por mulheres, assim, seguindo uma hierarquia social, a sobrecarrega de atividades nesse sexo é ainda maior, pois acabam assumindo excesso de trabalho, além da sua jornada doméstica (73,2\%) - cuidar da casa, da família e dos filhos (Tabela 2).

Tabela 2 - Características ocupacionais dos fibromiálgicos $(n=71)$.

\begin{tabular}{lcc}
\hline Variáveis & $\mathbf{n}$ & $\%$ \\
\hline Atividade remunerada & 44 & 62,0 \\
\hline Sim & 27 & 38,0 \\
Não & & \\
\hline Carga horária semanal & 58 & 81,7 \\
\hline 20 a 30 horas & 7 & 9,9 \\
36 a 40 horas & 2 & 2,8 \\
44 horas ou mais & & 35,8 \\
\hline Turno da atividade & 24 & 13,4 \\
\hline Não trabalha & 9 & 49,3 \\
Diurno e noturno & 33 & 1,5 \\
Diurno & 1 & \\
Noturno & & 73,2 \\
\hline Atividade doméstica & 52 & 26,8 \\
\hline Sim & 19 &
\end{tabular}

Fonte: Leite TO, et al., 2021.

Segundo Álvares TT e Lima MEA (2010), a sintomatologia inicial da fibromialgia ocorre por um episódio de estresse físico ou psicológico, prévio ou concomitante ao quadro. Dessa forma, alguns pacientes atribuem os sintomas à ergonomia inadequada no local de trabalho ou mesmo à atividade realizada. Constata-se um absenteísmo crescente nesses indivíduos ou mesmo a ausência de uma profissão pelo medo da piora ou exacerbação das manifestações clássicas da doença.

Em um estudo realizado por Filho GJA (2013), no qual teve a participação de 50 mulheres fibromiálgicas, ele relata que tarefas como limpar a casa, cuidar do quintal e jardim, foram as mais afetadas, uma vez que parcela significativa das pacientes nunca ou quase não conseguia realizá-las. Mulheres com fibromialgia acabam sendo fisicamente menos ativas do que mulheres saudáveis, pois as atividades diárias podem exacerbar à dor, mas mesmo assim uma parte delas não tem como se ausentar desas tarefas, seja por questões financeiras ou obrigação social.

O impacto no ciclo social do paciente com FM é visível e ocorre na medida em que afeta tanto as atividades diárias, quanto os seus relacionamentos. De acordo com o estudo de Oliveira JPR, et al. (2019a), a falta de entendimento e apoio dos membros da família, amigos e colegas de trabalho dos fibromiálgicos é frequente, pois se trata de uma patologia em que o quadro álgico é constante, impactando diretamente no desempenho da função. Tais fatores levam ao quadro de desânimo e falta de prazer para realizar atividades que exijam contatos e relações sociais e recreativas, tendendo ao isolamento.

Outro fator importante relacionado aos familiares é que aqueles que convivem mais intimamente com o paciente fibromiálgico pode desenvolver sintomas de ansiedade e depressão, pois acabam absorvendo 0 sofrimento que a doença proporciona ao seu parente (FILHO GJA, 2013).

Pacientes fibromiálgicos apresentam níveis mais altos de dor, o que acarreta limitações funcionais e físicas, menor flexibilidade, falta de condicionamento aeróbico, menor força e desempenho muscular, afetando diretamente a capacidade para realização das atividades de vida diária e ocupacionais (LORENA SB de, et al., 2016). Diante disso, observou-se a não realização de atividade física pela maioria desses indivíduos (36,6\%), como foi constatado na Tabela 3. 
Tabela 3 - Prática de atividade física dos fibromiálgicos $(n=71)$.

\begin{tabular}{lcc}
\hline Variáveis & $\mathbf{n}$ & $\%$ \\
\hline Realiza pelo menos $\mathbf{3 0}$ minutos & & 36,6 \\
\hline Não realiza & 26 & 31,0 \\
1 a 2x/semana & 22 & 29,6 \\
3 a 5x/semana & 21 & \\
\hline Atividade física & & 40,8 \\
\hline Corrida & 29 & 8,5 \\
Caminhada & 6 & 29,6 \\
Musculação & 21 & 9,9 \\
Ciclismo & 7 & 1,4 \\
Natação & 1 & 1,4 \\
Treino funcional & 1 & 1,4 \\
Dança & 1 & 2,8 \\
Outro & 2 &
\end{tabular}

Fonte: Leite TO, et al., 2021.

Para Matsudo SM e Lillo JLP (2019), os sintomas clássicos da fibromialgia, a dor e a fadiga, fazem com que os pacientes limitem, ou não realizem, atividade física diária e se tornem menos envolvidos em programas de exercícios físicos e atividades esportivas de qualquer tipo. Além disso, alguns indivíduos têm medo de que qualquer movimento possa aumentar os sintomas, evitando mais ainda a realização dessas práticas.

Observa-se benefícios das atividades físicas realizadas na água que, provavelmente, devido à temperatura da água e à sensação de prazer, podem melhorar a rigidez e dor muscular que limitam a tolerância dos exercícios realizados fora da água. A redução desses sintomas pode ser importante para melhorar a autoeficácia para o exercício, humor e sono, que contribuem diretamente para a melhora da qualidade de vida e o bem-estar de pessoas com FM (BIDONDE J, et al., 2017).

Em relação à musculação, é visível o seu papel para combater o excesso de peso, a sarcopenia e a perda funcional. Por isso, o ideal seria a prática da mesma pelos fibromiálgicos para manter ou melhorar a massa e a força muscular e, consequentemente, a composição corporal e a capacidade funcional (NELSON NL, 2015).

Alguns dos mecanismos para explicar a analgesia induzida pelo exercício incluem que a atividade física regular modula o sistema imune na dor local, além da sua atuação no SNC. Ocorre a redução da ativação da glia e das citocinas inflamatórias e elevação das citocinas anti-inflamatórias na medula espinhal. Dessa forma, o paciente precisa compreender a importância e adotar as práticas regulares de atividades corporais, as quais podem incidir positivamente na melhora da dor fisíca e dos quadros psíquicos, contribuindo para melhora do seu quadro geral (MATSUDO SM e LILLO JLP, 2019).

Segundo o Colégio Americano de Medicina Esportiva (ACMS), a frequência de um a dois dias por semana é o ideal, mas a diminuição dos sintomas é maior quando a frequência aumenta para três dias. É essencial deixar um tempo de recuperação entre os exercícios na mesma sessão e entre os dias de exercício, além de alterná-los nas diferentes partes do corpo e nos diferentes sistemas (cardiovascular e muscular). Entre as diretrizes do ACSM está a de que a progressão do exercício depende inteiramente dos sintomas do paciente. No presente estudo foi possível observar, entre os praticantes de alguma modalidade física, percentual considerável de alguma prática corporal, sendo a frequência de duas vezes por semana e acima prevalentes (MATSUDO SM e LILLO JLP, 2019).

Observa-se que diante da crise álgica outras questões são desencadeadas, como a dificuldade em se manter com autonomia, relacionado ao autocuidado, higiene pessoal, aparência e a própria autoestima, haja vista que os transtornos de humor estão presentes, afetando diretamente o bem-estar do paciente (OLIVEIRA JPR, et al., 2019b). Assim, foi corroborado por esse estudo que $49,3 \%$ dos entrevistados apresentam sintomas depressivos de intensidade moderada a grave (Tabela 4). 
Tabela 4 - Intensidade dos sintomas depressivos nos fibromiálgicos $(n=71)$

\begin{tabular}{lcc}
\hline Variáveis & $\mathbf{n}$ & $\%$ \\
\hline Intensidade de sintomas depressivos & & \\
\hline Mínima & 16 & 22,5 \\
Leve & 20 & 28,2 \\
Moderada & 27 & 38,0 \\
Grave & 8 & 11,3 \\
\hline
\end{tabular}

Fonte: Leite TO, et al., 2021.

Sabe-se que há uma associação entre fibromialgia e sintomas de depressão, e isso foi constatado no estudo de Cordeiro IM, et al. (2021), no qual os sintomas depressivos estavam presentes em $80 \%$ da população entrevistada $(n=60)$. A característica de dor difusa e crônica, muitas vezes incapacitante, manifestada pela FM corrobora para essas alterações psicológicas, visto que quadros de dor favorecem o surgimento de transtornos psíquicos, como por exemplo depressão, ansiedade, transtornos de personalidade. O estresse emocional, por sua vez, é um dos principais agentes desencadeadores de transtornos mentais e responsável por agravar ou precipitar esses sintomas álgicos podendo, assim, perpetuar o ciclo patológico de dor crônica.

Em outra ponta do espectro, observa-se uma percepção negativa do estado de saúde desses fibromiálgicos, haja vista que quem é acometido por uma síndrome dolorosa crônica apresenta postura evitativa e pessimista do seu próprio "eu" (CRUZ BD, 2020).

O estado psicológico também desempenha um papel muito importante na função sexual normal. Para Centurion NB (2017), as mulheres com fibromialgia apresentam menores escores de desejo sexual, satisfação sexual, orgasmo, excitação sexual, lubrificação vaginal, e sensação clitoriana, além das várias formas de depressão, baixa autoestima ou estresse emocional, o que torna difícil a comunicação com os seus parceiros. Assim, a doença crônica influencia nas relações íntimas entre marido e mulher, devido aos sintomas de dor, fadiga e incapacidade de se movimentar, o que gera uma tensão entre eles (MELO FABP, et al., 2014).

É conhecido que pacientes acima do peso apresentam mais dor musculoesquelética quando comparados com os que apresentam peso ideal. Acrescenta-se também que indivíduos acima do peso tendem a apresentar condições reumáticas como a osteoartrite de joelho $(\mathrm{OA})$, a síndrome do túnel do carpo e a própria fibromialgia. O sobrepeso ou obesidade estão comumente presentes em fibromiálgicos, podendo piorar o quadro clínico doloroso. Isso se deve principalmente à depressão, pois reduz o limiar de dor e piora a inatividade física, agravando a limitação funcional e, consequentemente, impactando na qualidade de vida (DIAS DNG, et al., 2017).

Outro fator que piora diretamente a depressão no paciente com fibromialgia são as queixas de sono, como insônia, sensação de cansaço ao andar, redução das horas de sono, aumento no número de interrupções do sono por noite, dificuldade em obter um sono restaurador e aumento da sonolência durante o dia, caracterizada por dificuldade em manter a vigília (AVILA S, et al., 2014). Estudos têm demonstrado que a severidade da doença, bem como a duração e a gravidade dos sintomas, podem impactar na qualidade de vida e originar essas alterações, assim como predizer a dor subsequente nesta população e exacerbar os sintomas (FERNANDES RCS, et al., 2020).

No presente estudo, foi observado que $84,5 \%(n=60)$ dos pacientes fazem algum tipo de tratamento, seja terapia farmacológica ou a não farmacológica, e 15,5\% ( $n=11)$ não utilizam alguma abordagem terapêutica. Dentre os fármacos questionados, 50,7\% $(n=36)$ afirmaram usar antidepressivos, e em sua maioria associados a no mínimo outras três classes de medicações $(29,6 \%)$. Quanto à terapia não farmacológica, $26,8 \%$ dos entrevistados relataram não fazer. Dos que fazem, apenas 16,9\% ( $n=12)$ realizam psicoterapia. Não foi perguntado o tipo de terapia especifica, mas, de acordo com a literatura, a TCC é a mais indicada nos fibromiálgicos. 
Os antidepressivos tricíclicos foram os primeiros fármacos a serem intensamente estudados para o tratamento da fibromialgia, sendo a amitriptilina a principal representante. $O$ tramadol é um opioide de ação central, aliviando a dor e baixando o seu limiar, e o seu uso está particularmente indicado nas fases de agudização da doença. A pregabalina, anticonvulsivante, mostrou-se efetivo na redução da severidade da dor, da fadiga e na melhoria da qualidade do sono. A melatonina é um hormônio secretado pela glândula pineal com propriedades reguladoras do sono. Além dessa propriedade, apresenta ações analgésicas, antiinflamatórias, antioxidantes, ansiolíticas e antidepressivas. Todo este potencial estimulou seu uso no manejo do quadro clínico da fibromialgia (GOMES CSSP, 2020).

A TCC é a abordagem comumente de escolha para atuação em fibromiálgicos, pois auxilia o pensamento e a forma pelo qual o paciente irá agir diante dos acontecimentos do cotidiano. Atribui-se à ela o preceito de que pensamentos interferem nos sentimentos mais do que qualquer elemento externo, por isso, procura-se exercitar habilidades para enfrentar o emaranhado emocional que implica veementemente em intensificar os sintomas da fibromialgia (POLIZEL IA, 2019).

Destarte, o tratamento da fibromialgia continua a ser um forte desafio para os profissionais de saúde, por conta do desconhecimento da sua etiologia. De certa forma, ele é individualizado e exclusivamente sintomático, objetivando a redução do sofrimento de seus portadores, a melhora da funcionalidade, e a promoção da autonomia pessoal. Além disso, deve ser reforçado que uma melhoria significativa dos sintomas raramente ocorre sem a participação ativa do fibromiálgico, sendo fundamental uma verdadeira aliança médico-paciente (OLIVEIRA JO e ALMEIDA MB, 2018).

\section{CONCLUSÃO}

O presente estudo evidenciou maior prevalência de fibromialgia entre as mulheres, com idade entre 31 a 50 anos, com elevado grau de escolaridade, que eram casadas ou viviam em união estável. Em relação a atividade laboral, a maioria dos participantes realizam atividade remunerada, com jornada semanal em torno de 20 a 30 horas e trabalhando, ao menos, em dois turnos. Evidenciou-se também um percentual elevado de depressão de moderada a grave entre os indivíduos respondentes, apontando a necessidade de ações direcionadas à saúde mental dos fibromiálgicos.

\section{REFERÊNCIAS}

1. ÁLVARES TT, LIMA MEA. Fibromialgia: interfaces com as LER/DORT e considerações sobre sua etiologia ocupacional. Ciência \& Saúde Coletiva, 2010; 15(3): 802-812.

2. ANDRADE ICS. Tratamento farmacológico e não farmacológico da fibromialgia: uma revisão. Monografia (Curso de Graduação em Farmácia) - Centro de Educação e Saúde. Universidade Federal de Campina Grande, Cuité, 2017; 61 p.

3. ARAUJO ABM. Narrativas de vida de mulheres com fibromialgia: autogerenciamento da dor crônica. Dissertação (Mestrado em Enfermagem). Universidade do Estado do Rio de Janeiro, Rio de Janeiro, 2020: $100 \mathrm{p}$.

4. AVILA S, et al. Caracterização dos padrões de dor, sono e alexitimia em pacientes com fibromialgia atendidos em um centro terciário brasileiro. Revista Brasileira de Reumatologia, 2014; 54(5): 409-413.

5. BECK AT, et al. Terapia cognitiva da depressão. Rio de Janeiro: Zahar, 1982; $419 \mathrm{p}$.

6. BIDONDE J, et al. Aerobic exercise training for adults with fibromyalgia. Cochrane Database of Systematic Reviews, 2017; 6: CD012700.

7. CASTRO SMJ, et al. Teoria da resposta ao item aplicada ao Inventário de Depressão Beck. Revista Brasileira de Epidemiologia, 2010; 13(3): 487-501.

8. CENTURION NB. A vivência da sexualidade em mulheres com fibromialgia: um estudo clínico-qualitativo. Dissertação (Mestrado em Psicologia Aplicada) - Instituto de Psicologia. Universidade Federal de Uberlândia, Uberlândia, 2017: $102 \mathrm{p}$.

9. CORDEIRO IM, et al. Avaliação da funcionalidade e a correlação com ansiedade e depressão em pacientes com fibromialgia atendidos no IMIP. Faculdade Pernambucana de Saúde. Recife, 2021; $25 p$.

10. CRUZ BD. Avaliação da Qualidade de Vida e Comprometimento Funcional em Pacientes com Fibromialgia. Monografia (Curso de Graduação em Medicina). Universidade Federal da Fronteira Sul, Passo Fundo, 2020; 83 p.

11. CUNHA JA. Manual da versão em português das ESCALAS BECK. São Paulo: Casa do Psicólogo, 2001. 
12. DIAS DNG, et al. Prevalência de fibromialgia em pacientes acompanhados no ambulatório de cirurgia bariátrica do Hospital de Clínicas do Paraná - Curitiba. Revista Brasileira de Reumatologia, 2017; 57(5): 425-430.

13. FARIA PC, et al. Fibromialgia: diagnóstico, fisiopatologia e tratamentos. Revista Conexão Ciência - UNIFOR-MG, 2014; 9(1): 01-19.

14. FERNANDES RCS, et al. Relação entre distúrbios do sono e severidade da fibromialgia: o impacto na qualidade de vida de brasileiras usuárias de redes sociais. Revista Brasileira de Qualidade de Vida, 2020; 12(1): 1-13.

15. FILHO GJA. Impacto da fibromialgia na qualidade de vida de pacientes assistidas no CRER, em Goiânia, Goiás. Dissertação (Mestrado em Ciências Ambientais e Saúde). Pontifícia Universidade Católica de Goiás, Goiânia, 2013; $80 \mathrm{p}$.

16. FRIEDRICH JV, et al. Fibromialgia: importância do conhecimento da doença e seus tratamentos. FAG journal of health, 2020; 2(2): 307-314.

17. GOMES CSSP. Fibromialgia: Etiologia, Diagnóstico e Tratamento. Dissertação (Mestrado em Ciências Farmacêuticas). Universidade Fernando Pessoa, Porto, 2020; 79 p.

18. GORESTEIN C, ANDRADE L. Inventário de depressão de Beck: propriedades psicométricas da versão em português. Revista de Psiquiatria Clínica, 1998; 25(5): 245-250.

19. HEYMANN RE, et al. Novas diretrizes para o diagnóstico da fibromialgia. Revista Brasileira de Reumatologia, 2017; $57(2): 467-476$.

20. LORENA SB, et al. Avaliação de dor e qualidade de vida de pacientes com fibromialgia. Revista Dor. São Paulo, 2016; 17(1): 8-11.

21. MATSUDO SM, LILLO JLP. Fibromialgia, atividade física e exercício: revisão narrativa. Revista Diagnóstico e Tratamento, 2019; 24(4): 174-182.

22. MELO FABP, et al. Relação entre qualidade de vida e sexualidade em mulheres fibromiálgicas. Revista de enfermagem UFPE on line, 2014; 8(2): 3587-3593.

23. MELO GA, et al. Análise dos perfis sociodemográfico e clínico de mulheres com fibromialgia. Temas em saúde, 2019; 19(5): 24-34.

24. MILANI RG, et al. A dor psíquica na trajetória de vida do paciente fibromiálgico. Aletheia, 2012; 38-39: 55-66.

25. NELSON NL. Muscle strengthening activities and fibromyalgia: a review of pain and strength outcomes. Journal of bodywork and movement therapies, 2015; 19(2): 370-376.

26. OLIVEIRA JO, ALMEIDA MB. The current treatment of fibromyalgia. Brazilian Journal of Pain, 2018; 1(3): $255-262$.

27. OLIVEIRA JPR, et al. Qualidade de vida e autocuidado de mulheres que vivem com fibromialgia: uma revisão integrativa. Revista Nursing, 2019b; 22(251): 2880-2886.

28. OLIVEIRA JPR, et al. O cotidiano de mulheres com fibromialgia e o desafio interdisciplinar de empoderamento para o autocuidado. Revista Gaúcha de Enfermagem, 2019a.

29. POLIZEL IA. O tratamento da Fibromialgia por meio da Terapia Cognitivo-Comportamental. Monografia (Especialização em Terapia Cognitivo-Comportamental). Centro de Estudos em Terapia Cognitivo-Comportamental, São Paulo, 2019; 32 p.

30. RAMIRO FS, et al. Investigação do estresse, ansiedade e depressão em mulheres com fibromialgia: um estudo comparativo. Revista Brasileira de Reumatologia, 2013; 54(1): 27-32.

31. RODRIGUES GF, et al. A relação entre fibromialgia e depressão. Monografia (Curso de Graduação em Psicologia). Faculdade Sant'Anna, São Paulo, 2016: 18 p.

32. SOUZA JB, PERISSINOTTI DMN. A prevalência da fibromialgia no Brasil: estudo de base populacional com dados secundários da pesquisa de prevalência de dor crônica brasileira. Brazilian Journal of Pain, 2018; 1(4): 345-348. 